\title{
A CD4 T cell gene signature for early rheumatoid arthritis implicates interleukin 6-mediated STAT3 signalling, particularly in anti-citrullinated peptide antibody-negative disease
}

\author{
Arthur G Pratt, ${ }^{1,2}$ Daniel C Swan, ${ }^{3}$ Sarah Richardson, ${ }^{1}$ Gillian Wilson, ${ }^{2}$ \\ Catharien M U Hilkens, ${ }^{1}$ David A Young, ${ }^{1}$ John D Isaacs ${ }^{1,2}$
}

- Additional data are published online only. To view these files please visit the journal online (http://ard.bmj.com/content/ early/recent).

${ }^{1}$ Institute of Cellular Medicine (Musculoskeletal Research Group), Newcastle University, Newcastle upon Tyne, UK 2Musculoskeletal Directorate, Newcastle upon Tyne Hospitals NHS Trust, Newcastle upon Tyne, UK

3/nstitute of Cell and Molecular Biology (Bioinformatics Support Unit), Newcastle University, Newcastle upon Tyne, UK

\section{Correspondence to}

Professor John D Isaacs, Newcastle University, Institute of Cellular Medicine (Musculoskeletal Research Group), Newcastle upon Tyne NE2 4HH, UK;

j.d.isaacs@ncl.ac.uk

Accepted 23 February 2012

\section{ABSTRACT}

Objective We sought clinically relevant predictive biomarkers present in CD4 T-cells, or in serum, that identified those patients with undifferentiated arthritis (UA) who subsequently develop rheumatoid arthritis (RA). Methods Total RNA was isolated from highly purified peripheral blood CD4 T cells of 173 early arthritis clinic patients. Paired serum samples were also stored. Microarray analysis of RNA samples was performed and differential transcript expression among 111 'training cohort' patients confirmed using real-time quantitative PCR. Machine learning approaches tested the utility of a classification model among an independent validation cohort presenting with UA (62 patients). Cytokine measurements were performed using a highly sensitive electrochemiluminescence detection system.

Results A 12-gene transcriptional 'signature' identified RA patients in the training cohort and predicted the subsequent development of RA among UA patients in the validation cohort (sensitivity $68 \%$, specificity 70\%). STAT3-inducible genes were over-represented in the signature, particularly in anti-citrullinated peptide antibody-negative disease, providing a risk metric of similar predictive value to the Leiden score in seronegative UA (sensitivity $85 \%$, specificity $75 \%$ ). Baseline levels of serum interleukin 6 (IL-6) (which signals via STAT3) were highest in anti-citrullinated peptide antibodies-negative RA and distinguished this subgroup from non-RA inflammatory synovitis (corrected $p<0.05$ ). Paired serum IL-6 measurements correlated strongly with STAT3-inducible gene expression.

Conclusion The authors have identified IL-6-mediated STAT-3 signalling in CD4 T cells during the earliest clinical phase of RA, which is most prominent in seronegative disease. While highlighting potential biomarker(s) for early $\mathrm{RA}$, the role of this pathway in disease pathogenesis awaits clarification.

The importance of prompt disease-modifying therapy in early rheumatoid arthritis (RA) is now established. ${ }^{12}$ Yet about $40 \%$ of patients with new-onset inflammatory arthritis present with disease that is unclassifiable at inception, having a so-called undifferentiated arthritis (UA).3 Timely intervention for the subset of these UA patients who subsequently develop RA therefore remains problematic. The issue is highlighted by the publication of updated
RA classification criteria ${ }^{4}$ and a validated 'prediction rule' that foretells risk of UA progression to RA. ${ }^{5}$ Such approaches rely heavily on autoantibody status, emphasising the specificity of circulating anti-citrullinated peptide antibodies (ACPA) for RA. ${ }^{6}$ Consequently, the diagnosis of ACPAnegative RA remains challenging in the early arthritis clinic (EAC), being frequently delayed despite application of the prediction rule. ${ }^{7}$

The potential for the whole-genome transcription profiling to yield clinically relevant prognostic 'gene signatures' in autoimmune disease has been demonstrated. ${ }^{89}$ Applying a similar, prospective approach to the discovery of predictive biomarkers in UA should complement existing diagnostic algorithms, while providing new insights into disease pathogenesis. ${ }^{10}$ However, the use of peripheral blood (PB) mononuclear cells for transcriptional analysis may result in data that are biased by relative subset abundance. ${ }^{11}$ To address this, protocols for rapid ex vivo positive selection of cell subsets for the purpose of transcription profiling have been validated. ${ }^{12}$ Although no single cell-type is exclusively implicated in RA, many of its established and emerging genetic associations implicate the CD4 $\mathrm{T}$ cell. ${ }^{13}$ We therefore hypothesised that the PB CD4 T-cell transcriptome would provide a useful substrate for both biomarker discovery and a pathophysiological understanding of RA induction.

\section{MATERIALS AND METHODS}

A complete description of experimental and bioinformatics approaches are given in the online supplementary text.

\section{Patients}

Patients with recent onset arthritis, naive to disease-modifying anti-rheumatic drugs and corticosteroids, were recruited between September 2006 and December 2008. An initial working clinical diagnosis was updated by the consulting rheumatologist at consecutive clinic visits for the duration of the study-median 28 months and $>12$ months in all cases. RA was diagnosed only where 1987 American College of Rheumatology classification criteria $^{14}$ were fulfilled; UA was defined as a 'suspected inflammatory arthritis where RA remained a possibility, but where established classification criteria for any rheumatological condition remained 
Table 1 Clinical characteristics of the rheumatoid arthritis (RA) and non-RA comparator groups used to generate list of differentially expressed genes, which together comprise a training cohort for machine-learning (total $n=111$ ) and the independent undifferentiated arthritis (UA) validation cohort $(n=62)$

\begin{tabular}{|c|c|c|c|c|}
\hline & \multicolumn{3}{|c|}{ Training cohort } & \multirow{2}{*}{$\begin{array}{l}\text { Test cohort } \\
\text { UAt }(n=62)\end{array}$} \\
\hline & $\mathrm{RA}(\mathrm{n}=47)$ & Non-RA $(n=64)$ & $\mathbf{p}^{*}$ & \\
\hline Age (years; mean, SD range) & $60(46-74)$ & $48(34-62)$ & 0.01 & $52(37-67)$ \\
\hline$\%$ Female & 65 & 61 & NS & 77 \\
\hline \% White Caucasian & 96 & 92 & NS & 90 \\
\hline Symptom duration (weeks; median, IQR) & $12(8-24)$ & $21(10.5-52)$ & 0.026 & $14(12-26)$ \\
\hline Tender joint count (median, IOR) & $10(4-15)$ & $7(2-14)$ & NS & $8(3-16.5)$ \\
\hline Swollen joint count (median, IQR) & $6(2-10)$ & $0(0-2)$ & $<0.001$ & $1(0-3)$ \\
\hline Morning stiffness (hours; median, IQR) & $1(0.75-2)$ & $0.75(0.1-2)$ & 0.007 & $1(0.5-2)$ \\
\hline ESR (s; median, IOR) & $56(30-78)$ & $24(14-52)$ & $<0.001$ & $30(18-60)$ \\
\hline CRP (g/l; median, IQR) & $17(9-62)$ & $5(2.5-19)$ & $<0.001$ & $8.5(0-17)$ \\
\hline ACPA+ (number; per cent) & $29(62)$ & $0(0)$ & $<0.001$ & $13(21)$ \\
\hline $\mathrm{RF}+$ (number; per cent) & $36(77)$ & $3(6)$ & $<0.001$ & $20(32)$ \\
\hline DAS28 (median) & 5.37 & NA & - & \\
\hline Leiden prediction score (median, IQR) & NA & NA & - & $6.4(5-7.6)$ \\
\hline \multicolumn{5}{|l|}{ Outcome diagnoses (number, per cent) } \\
\hline RA & $47(100)$ & $0(0)$ & - & $25(40)$ \\
\hline Seronegative sponyloarthropathy & - & $22(34)$ & - & $8(13)$ \\
\hline Self-limiting inflammatory & - & $12(19)$ & - & $9(15)$ \\
\hline Other inflammatory & - & $3(5)$ & - & $2(3)$ \\
\hline $\mathrm{OA} /$ non-inflammatory & - & $27(42)$ & - & $18(29)$ \\
\hline
\end{tabular}

unmet' (see online supplementary text and supplementary table S1). Individuals whose arthritis remained undifferentiated at the end of the study were excluded. Patients gave written informed consent before inclusion into the study, which was approved by the local regional ethics committee.

\section{CD4 T-cell RNA processing and array analysis}

Whole blood drawn between 13:00 and 16:30 was stored at room temperature for $\leq 4 \mathrm{~h}$ before processing. After monocyte depletion by immuno-rosetting, an automated magnetic beadbased positive selection protocol was used to isolate CD4 cells (Stemcell Technologies, Vancouver, Canada). Using this approach, a median CD4 T-cell purity of $98.9 \%$ was achieved (range 95-99.7\%), which was determined using flow cytometry (see online supplementary figure S1). Total CD4 T-cell RNA was immediately extracted, then quality controlled using an Agilent 2100 Bioanalyzer (Agilent, Santa Clara, California, USA). The median RNA integrity number ${ }^{15}$ of samples used was 9.4. cRNA generated from $250 \mathrm{ng}$ total RNA (Illumina TotalPrep RNA Amplification Kit) was hybridised to the Illumina Whole Genome 6v3 BeadChip (Illumina, San Diego, California, USA), representing 48804 known genes and expressed sequence tags. Array data were processed using Illumina BeadStudio software, then it was normalised, batch corrected, ${ }^{16}$ filtered and quality controlled as described (online supplementary text and figure S2).

To define differential expression a fold-change cut-off of 1.2 between comparator groups was combined with a significance level cut-off of $\mathrm{p}<0.05$ (Welch's $t$-test), corrected for multiple testing using the false-discovery-rate method of Benjamini et al. ${ }^{17}$ Genes thereby identified were used to train a support vector machine (SVM) classification model based on known outcomes among a 'training' sample set. ${ }^{18}$ The model's accuracy as a prediction tool was then assessed among an independent 'validation' sample set. To obtain larger lists of differentially expressed genes for biological pathway analysis, significance thresholds were relaxed through the omission of multipletest-correction and Ingenuity Pathways Analysis software (Ingenuity Systems, Redwood City, California, USA) was then employed.

\section{Serum cytokine measurement}

Between 13:00 and 16:30, baseline serum was drawn and frozen at $-80^{\circ} \mathrm{C}$ until use. Serum interleukin 6 (IL-6), soluble IL- 6 receptor (sIL6R), tumour necrosis factor $\alpha$ (TNF $\alpha$ ), leptin and granulocyte colony stimulating factor concentrations were measured using a highly sensitive electrochemiluminescence immunosorbance detection system (Meso Scale Discovery, Gaithersberg, Maryland, USA), assays having been validated as outlined (online supplementary text and figure S3).

\section{Quantitative real-time PCR}

CD4 T cell total RNA samples were reverse transcribed using superscript II reverse transcriptase and random hexamers, according to the manufacturer's instructions (Invitrogen, Carsbad, California, USA). Real-time PCR reactions were performed as part of a custom-made TaqMan Low Density Array (7900HT real-time PCR system, Applied Biosystems, Foster City, California, USA). Raw data were normalised and expressed relative to the housekeeping gene $\beta$-actin as $2-\Delta \mathrm{Ct}$ values. ${ }^{19}$

\section{General statistics}

Parametric and non-parametric analyses of variance, MannWhitney U tests, Pearson's correlation coefficients, intraclass correlations, multivariate analyses and the construction of receiver operator characteristic (ROC) curves were performed, as described, using SPSS version.15.0 (SPSS, Chicago, Illinois, USA). The derivation of Leiden prediction rules ${ }^{5}$ and 
Table 2 Fold-change and significance level for genes differentially expressed at inception among peripheral blood CD4 T cells between early arthritis clinic patients with inception diagnoses of RA and non-RA (confirmed at $\geq 1$ year; median 28 months follow-up). The official gene symbol and RefSeq accession number are given as identifiers. Listed STAT3-regulated genes are italicised. 12 genes included in statistically most robust 'RA signature' appear in boldface, and additional STAT3-regulated genes referred to in text are also provided

\begin{tabular}{|c|c|c|c|c|c|}
\hline \multirow[b]{2}{*}{$\begin{array}{l}\text { Gene (Accn. No.) } \\
\text { 12-Gene RA Signature: }\end{array}$} & \multicolumn{3}{|c|}{$\begin{array}{l}\text { Microarray data } \\
\text { (47 RA vs } 64 \text { non-RA) }\end{array}$} & \multicolumn{2}{|c|}{$\begin{array}{l}\text { qRT-PCR data } \\
\text { (32 RA vs } 41 \\
\text { non-RA*) }\end{array}$} \\
\hline & FC & $\begin{array}{l}\text { Uncorr. } \\
\text { pt }\end{array}$ & $\begin{array}{l}\text { Corr. } \\
\text { pt }\end{array}$ & FC & $\mathrm{p} \neq$ \\
\hline BCL3 (NM_005178) & 1.59 & $2.6 \times 10^{-5}$ & 0.03 & 2.15 & 0.005 \\
\hline SOCS3 (NM_003955) & 1.55 & $3.4 \times 10^{-6}$ & 0.03 & 1.83 & 0.002 \\
\hline PIM1 (NM_002648) & 1.52 & $6.8 \times 10^{-6}$ & 0.03 & 1.67 & 0.001 \\
\hline SBNO2 (NM_014963) & 1.47 & $1.2 \times 10^{-5}$ & 0.03 & 1.13 & 0.158 \\
\hline LDHA (NM_005566) & 1.23 & $3.8 \times 10^{-5}$ & 0.04 & 1.25 & 0.003 \\
\hline CMAH (NR_002174) & 1.2 & $1.7 \times 10^{-5}$ & 0.03 & 1.40 & 0.003 \\
\hline NOG (NM_005450) & -1.32 & $3.1 \times 10^{-5}$ & 0.03 & -1.59 & 0.004 \\
\hline PDCD1 (NM_005018) & 1.42 & $1.0 \times 10^{-5}$ & 0.03 & ND & ND \\
\hline IGFL2 (NM_001002915) & 1.31 & $1.1 \times 10^{-7}$ & 0.002 & ND & ND \\
\hline LOC731186 (XM_001128760) & 1.28 & $2.3 \times 10^{-5}$ & 0.03 & ND & ND \\
\hline MUC1 (NM_001044391) & 1.26 & $2.0 \times 10^{-5}$ & 0.03 & ND & ND \\
\hline GPRIN3 (CR743148)§ & 1.32 & $2.1 \times 10^{-4}$ & 0.049 & ND & ND \\
\hline \multicolumn{6}{|l|}{ Additional STAT3-regulated: } \\
\hline ID3 (NM 002167) & -1.3 & $5.2 \times 10^{-4}$ & 0.16 & ND & ND \\
\hline MYC (NM̄ 002467) & 1.2 & 0.04 & 0.75 & 1.29 & 0.01 \\
\hline
\end{tabular}

*Baseline characteristics of Quantitative real-time PCR validation sub-cohort are similar to those of the training cohort overall (table 1) and are given in the online supplementary table S4.

†Calculations based on normalised expression values of array data; Welch's t-test, raw and multiple-test-corrected $\mathrm{p}$ values given (see methods).

$\ddagger$ Calculations based on expression data normalised to the house-keeping gene $\beta$-actin (2- $\Delta$ Ct): Mann-Whitney U test (see methods).

$\S$ Transcript CR743148 (Illumina Probe ID 6370082) has been retired from NCBI, but the expressed sequence tag corresponds to splice variant(s) within the GPRIN3 gene (chromosome 4.90).

$\mathrm{FC}$, linearised fold-change expression in RA relative to non-RA (ie, negative values represent genes downregulated in RA relative to non-RA by $v$-fold); ND, not done; $\mathrm{RA}$, rheumatoid arthritis.

transcriptional 'risk metrics' for ACPA-negative RA is outlined in the online supplementary text.

\section{RESULTS}

\section{Patient groups}

A total of 173 patient samples were retrospectively selected for microarray analysis. One hundred and eleven of these originated from patients assigned definitive diagnoses at inception, confirmed at a median 28 months follow-up (minimum 1 year); an RA versus non-RA discriminatory 'signature' was derived from this 'training cohort' alone. The remaining 62 samples, all representing UA patients, formed an independent 'validation cohort' for testing the utility of the 'signature' according to diagnostic outcomes as they evolved during the same follow-up period. As expected, the characteristics of the UA cohort (age, acute phase response, joint counts, etc.) fell between the equivalent measurements in the RA and control sample sets within the training cohort (table 1). For subsequent pathway analysis, all 173 samples were pooled before being divided into four categories based on diagnostic outcome at the end of the study (see online supplementary table S2).

\section{RA transcription 'signature' most accurate in ACPA-negative UA}

Using a significance threshold robust to multiple test correction (false-discovery-rate $\mathrm{p}<0.05),{ }^{17} 12$ genes were shown to be differentially expressed ( $>1.2$-fold) in PB CD4 T cells between 47 'training cohort' EAC patients with a confirmed diagnosis of $\mathrm{RA}$, and 64 who could be assigned non-RA diagnoses (table 2).
An extended list, obtainable by omitting multiple-test correction, appears as online supplementary gene-list 1. Supervised hierarchical cluster analysis of the resultant dataset (111 samples, 12 genes), demonstrated a clear tendency for EAC patients diagnosed with RA to cluster together based on this transcription profile (figure 1A). Quantitative real-time PCR (qRT-PCR) was used to analyse expression of seven of the differentially expressed genes in a subset of 73 samples (for baseline characteristics of this subset, see online supplementary table S4). Despite the reduced power to detect change in this smaller dataset, robust differential expression was confirmed for six of the seven genes (table 2).

To derive a metric denoting risk of progression to RA, the sum of normalised expression values for the 12-gene RA 'signature' was calculated for each individual in the training cohort (see online supplementary text). A ROC curve was constructed for this risk metric, the area under which (0.85; SEM=0.04) suggested promising discriminatory utility (figure 1B). A SVM based on the training cohort dataset was then applied to classify members of the validation cohort, correctly identifying UA patients who developed RA with a sensitivity, specificity, positive and negative likelihood ratio $(0.68,95 \%$ CI 0.48 to 0.83$)$; $0.70,95 \%$ CI0.60 to 0.87$) ; 2.2,95 \%$ CI 1.2 to 3.8 ) and $0.495 \%$ CI 0.2 to 0.8$)$, respectively. However, we observed that of the 13 ACPA-positive UA patients, 12 progressed to RA, indicating that autoantibody status alone was a more sensitive predictor of RA in this subset. By contrast, when applied exclusively to the ACPA-negative subset of the UA validation cohort $(n=49)$, the SVM classification model provided a sensitivity of 0.85 (95\% CI 0.58 to 0.96$)$ and a specificity of 0.75 (0.59-0.86) for progression to RA, thereby performing best in this diagnostically most challenging patient group. Hierarchical clustering of the ACPA-negative UA samples based on their 12-gene RA 'signature' expression profiles further illustrates molecular similarities within the ACPA-negative RA outcome group (figure 1C).

\section{Gene signature adds value to existing tools in diagnosing ACPA-negative UA}

Next, we tested the value of our 12-gene signature in comparison with the existing 'Leiden prediction rule' as a predictor of RA among UA patients. ${ }^{5}$ While the discriminatory utility achieved by the prediction rule in our UA cohort was comparable with that previously reported ( $\mathrm{n}=62 ; \mathrm{AU}$ ROC curve $=0.86$; $\mathrm{SEM}=0.05$, data not shown), its performance diminished among the ACPAnegative sub-cohort ( $\mathrm{n}=49$; $\mathrm{AU}$ ROC curve=0.74; $\mathrm{SEM}=0.08$; figure 1D). Employing a 12-gene risk metric, as described above, equivalent discriminatory utility was found in this sub-cohort (AU ROC curve=0.78; SEM=0.08, data not shown). However, by deriving a modified risk metric, which combined all features of the Leiden prediction rule with our 12-gene risk metric (see online supplementary text and table S5), and applying it to the independent ACPA-negative UA cohort, we could improve the utility of the prediction rule for seronegative UA patients (AU $\mathrm{ROC}=0.84$; SEM=0.06; figure 1D).

\section{STAT3 transcription profile is most prominent in ACPA-negative RA}

All 173 patients studied were now grouped into four categories based on outcome diagnosis alone: ACPA-positive RA, ACPA-negative RA, inflammatory non-RA controls and osteoarthritis(OA); their demographic and clinical characteristics are presented for comparison (online supplementary table 

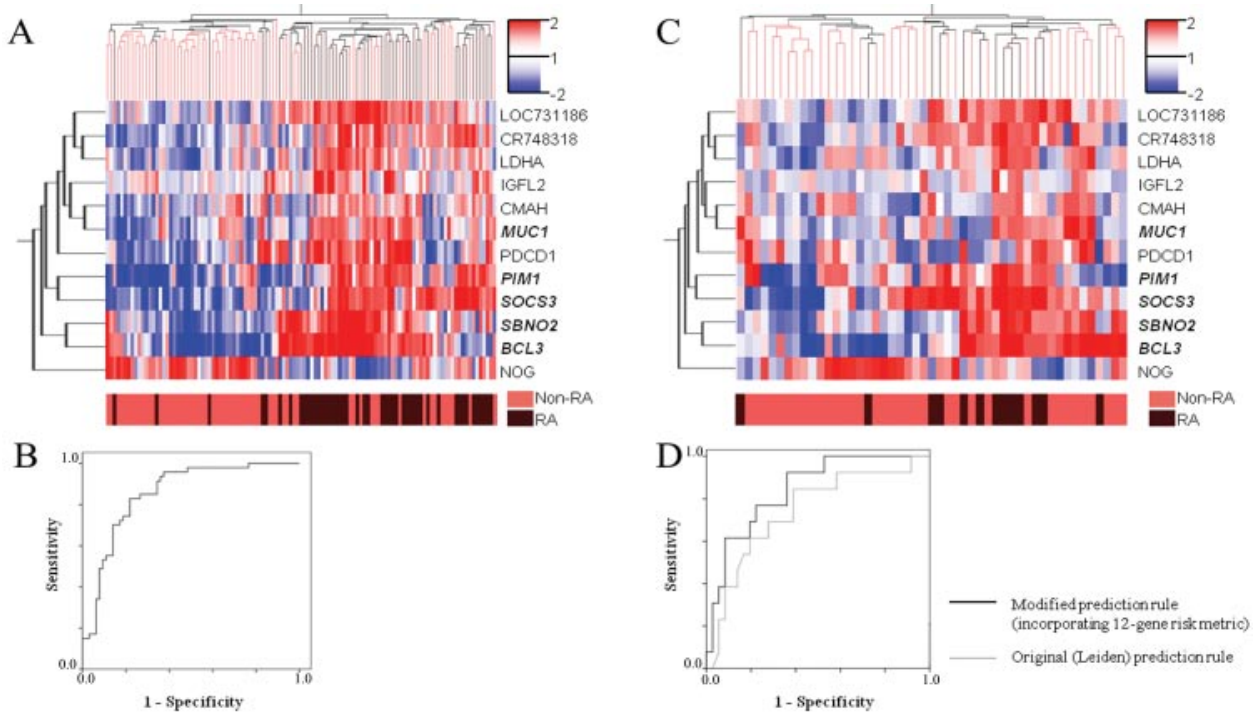

Figure 1 (A) Hierarchical clustering of training set: 111 samples represented by columns, and indicated individual genes by rows (italicised genes are STAT3 targets). Colour at each co-ordinate indicates gene-wise fold-expression relative to median (colour scale upper right). Underlying colour bar labels samples by inception diagnosis. (B) Receiver operator characteristic (ROC) plot for a rheumatoid arthritis (RA) risk metric derived from normalised gene expression values in the training cohort. Area under curve $=0.85$; $S E M=0.04 ; p<0.001$. (C) Hierarchical clustering of anticitrullinated peptide antibodies -negative undifferentiated arthritis 'validation' sub-cohort samples based on expression patterns of the same genes (interpretation as for figure 1A). (D) ROC curves comparing discriminatory value, in anti-citrullinated peptide antibodies-negative undifferentiated arthritis, of Leiden prediction rule (grey line) with a modified metric incorporating the 12-gene signature. Modified metric confers added value: area under $\mathrm{ROC}$ curve (original Leiden prediction rule) $=0.74 ; \mathrm{SEM}=0.08$ versus area under $\mathrm{ROC}$ curve (modified metric incorporating gene signature $)=0.84 ; \mathrm{SEM}=0.06 ; \mathrm{p}<0.001$ in both cases.

S2). Three lists of differentially expressed genes were then generated by comparing each of the 'inflammatory' groups (which themselves exhibited comparable acute phase responses) with the OA group ( $>1.2$-fold change; uncorrected $\mathrm{p}<0.05$; online supplementary gene-lists 2-4). The three lists were overlapped on a Venn diagram (figure 2).

A highly significant over-representation of genes involved in the cell cycle was identified in association with ACPA-positive RA (24/43; $\left.\mathrm{p}<1.0 \times 10^{-5}\right)$; figure 2; online supplementary genelist 5). In addition, genes involved in the regulation of apoptosis were over-represented in ACPA-negative RA patients, and RA was, in general, characterised by genes with functional roles in $T$ cell differentiation (figure 2 online supplementary gene-lists 5-8). Importantly, within the highly significant 12-gene RA 'signature,' several genes (PIM1, SOCS3, SBNO2, BCL3 and MUC1) were noted to be STAT3-inducible based on literature sources. ${ }^{20-25}$ The majority of these were more markedly differentially expressed in ACPA-negative than ACPA-positive RA (figures $3 \mathrm{~A}, \mathrm{~B}$ and online supplementary figures $\mathrm{S} 4 \mathrm{~A}-\mathrm{C}$ ). Additional STAT3-inducible genes (MYC, IL2RA) 202627 exhibited similar expression patterns, and there was a trend for STAT3 to be upregulated in ACPA-negative compared with ACPA-positive RA (online supplementary figures S4D-F). Moreover, a reciprocal pattern of expression across outcome groups was observed for the dominant negative helix-loop-helix protein-encoding gene inhibitor of DNA-binding 3 (ID3) (online supplementary figure S4G), consistent with its putative regulatory role in STAT3 signalling. ${ }^{28} \mathrm{MYC}$ and ID3, although absent from the discriminatory RA signature under the stringent significance thresholds used, were however robustly differentially expressed between RA and non-RA patients within the training cohort (table 2). Finally, in relation to both the 12-gene signature and the extended list of genes exclusively deregulated in ACPA-negative RA (online supplementary gene list 6), overlap with independently predicted STAT3-inducible gene sets (see online supplementary text and supplementary gene list 9) confirmed a preponderance of STAT3-inducible genes (hypergeometric $p$-values $<0.005$ in both cases; see online supplementary text) - which was not seen for genes deregulated only in ACPApositive RA ( $p=0.19)$.

\section{Serum IL-6 is highest in ACPA-negative RA and independently predicts CD4 STAT3-inducible gene expression}

Since one classical mechanism of STAT3 phosphorylation is via gp130 co-receptor ligation, ${ }^{29}$ we hypothesised that increased systemic levels of a key gp130 ligand and pro-inflammatory cytokine, IL-6, may be responsible for the STAT3-mediated transcriptional programme in early RA patients. Baseline serum IL- 6 was measured in 131 of the 173 EAC patients which were subsequently grouped according to their ultimate diagnosis (ACPA-negative RA, ACPA-positive RA, non-RA inflammatory arthropathy or OA). IL-6 levels were low overall (generally $<100 \mathrm{pg} / \mathrm{ml}$ ), but were highest in the ACPA-negative RA group (figure $3 \mathrm{C}$ ). Indeed, unlike the generic marker of systemic inflammation $\mathrm{C}$ reactive protein (CRP), baseline IL- 6 discriminated ACPA-negative RA from non-RA inflammatory arthritides (figures $3 \mathrm{C}, \mathrm{D}$ ). Furthermore, among individuals for whom paired and contemporaneous serum IL-6 and PB CD4 T-cell RNA samples were available, clear correlations between IL- 6 and the normalised expression of STAT3-inducible genes were seen (figures 4A-D; also online supplementary figures S5A-D); for example, serum IL-6 measurements correlated with normalised SOCS3 expression: Pearson's $\mathrm{R}=0.57, \mathrm{p}<0.001$ (figure 4A). Multivariate analysis confirmed that IL-6, but not CRP or TNF $\alpha$ (which does not signal via STAT3), independently predicted PB CD4 T cell SOCS3 expression ( $\beta=0.53 ; p<0.001$; see online supplementary table $\mathrm{S} 6$ ) excluding a more general influence of inflammation. 


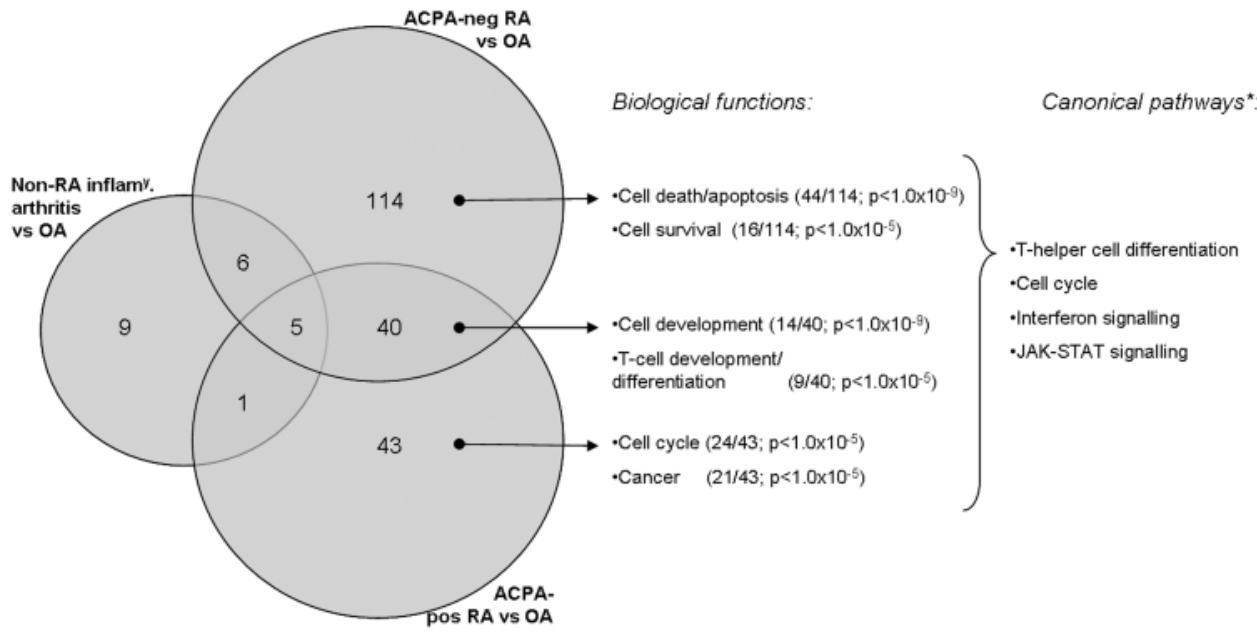

Figure 2 Functional analysis of array data. Genes differentially expressed $(>1.2$-fold change; $p<0.05)$ between osteoarthritis $(0 \mathrm{~A})$ and three separate inflammatory comparator groups were overlapped in a Venn diagram (see text, and online supplementary gene lists 2-4, for detailed list compositions). Genes uniquely deregulated in rheumatoid arthritis (RA) (anti-citrullinated peptide antibodies (ACPA)-negative, ACPA-positive or both) could thereby be identified and subjected to pathway analysis (see text). The top two over-represented biological functions identified for the three indicated sets are shown, along with the proportion of the set associated with the function in question, and a $\mathrm{p}$ value relating to the likelihood of given proportions occurring by chance (Fisher's exact test). Online supplementary gene lists 5-7 summarise functionally related genes thereby identified. The three indicated sets were combined to identify canonical pathways over-represented among genes differentially expressed between RA and $\mathrm{OA}$ in general. Pathways of particular interest in the biological context are listed (genes in question are listed in online supplementary gene list 8), *hypergeometric $p$-values (Fisher's exact) in each case $<0.01$.

Given that only $30-50 \%$ of $\mathrm{PB}$ CD4 T cells are thought to express membrane-bound IL6R, ${ }^{30}$ we also measured sIL6R (as a surrogate of IL-6R trans-signalling) ${ }^{31}$ and two other gp130 ligands, granulocyte colony stimulating factor and leptin, both of which have been implicated in RA pathogenesis. ${ }^{32} 33$ However, levels in sera from a subset of 80 study patients correlated with neither the diagnostic outcome nor the STAT3 gene expression. Finally, IL-10 and IL-17, which are both STAT3 activators, ${ }^{34}$ were undetectable in the vast majority of sera (data not shown).

\section{STAT3-inducible, RA-associated expression signature is activated by IL-6 in primary CD4 T cells of healthy donors in vitro} To confirm that the observed deregulated expression of STAT3 target genes among early RA patients was downstream of IL- 6 signalling, primary human CD4 $\mathrm{T}$ cells were incubated in vitro with recombinant human IL- 6 and the expression of relevant target genes measured at 1 and $6 \mathrm{~h}$ (see online supplementary text and figures S6-S7). Robust upregulation of SOCS3, PIM1, BCL3 and MYC was observed consistently $1 \mathrm{~h}$ after the addition of IL-6. A similar trend was seen for SBNO2, which became significant in the presence of recombinant soluble human IL-6 receptor. Conversely and consistent with prior observations, a distinct trend towards repression of ID3 was seen in response to IL-6 plus sIL6R.

\section{DISCUSSION}

We present a unique analysis of the CD4 T-cell transcriptome in a well-characterised inception cohort of early arthritis patients attending a routine EAC in UK. As a potential diagnostic tool, it is significant that our 12-gene 'RA expression signature' (table 2) performed best among the diagnostically challenging ACPA-negative UA patient group. Intriguingly, these findings support the involvement of CD4 $\mathrm{T}$ cells in both ACPA positive and negative disease.
The signature's sensitivity and specificity $(0.85$ and 0.75$)$ for predicting subsequent RA in seronegative UA patients equate to a positive likelihood ratio of 3.4, indicating that a prior probability of $25 \%$ for RA progression among this cohort (13 of the 49 patients progressed to RA) doubles to $53 \%$ for an individual who has been assigned a positive SVM classification. ${ }^{35}$ Moreover, of the 13 ACPA-negative UA patients who progressed to RA in our cohort, 8 fell into an 'intermediate' risk category for RA progression according to the validated Leiden prediction score. ${ }^{5}$ Encouragingly, all but one of these patients were correctly classified based on their 12-gene expression profile. Our proposal that this approach might add value to existing algorithms for the diagnosis of ACPA-negative UA is further supported by the construction of ROC curves comparing the Leiden prediction rule with a modified risk metric that incorporates features of our gene signature (figure 1D).

Our data indicate that PB CD4 $\mathrm{T}$ cells in early RA are characterised by a predominant upregulation of biological pathways involved in cell cycle progression (ACPA-positive) and survival, death and apoptosis (ACPA-negative) (figure 2; also online supplementary gene lists 5-6). Pathway analysis also suggested that T-cell development and differentiation were deregulated in both RA serotypes (online supplementary gene list 7). These findings concur with previous observations of impaired T-cell homeostasis in RA, characterised by increased turnover, telomere shortening and immunosenescence. ${ }^{36}{ }^{37}$ Given the well-characterised importance of the STAT3 signalling pathway in both oncogenesis and T-cell survival, it was notable that five genes from our statistically robust 12-gene RA signature are downstream of STAT3 signalling. ${ }^{20-25}$ The degree to which these genes sub-cluster according to the expression pattern among individuals in both the training and validation cohorts (figure $1 \mathrm{~A}, \mathrm{C}$ ) presumably reflects their co-regulation by STAT3. Their upregulation was generally most pronounced in ACPAnegative RA (figure $3 \mathrm{~A}, \mathrm{~B}$; also online supplementary figure $\mathrm{S} 4 \mathrm{~A}-\mathrm{C})$, explaining why the predictive utility of the 12-gene signature was optimal in this disease subset. 

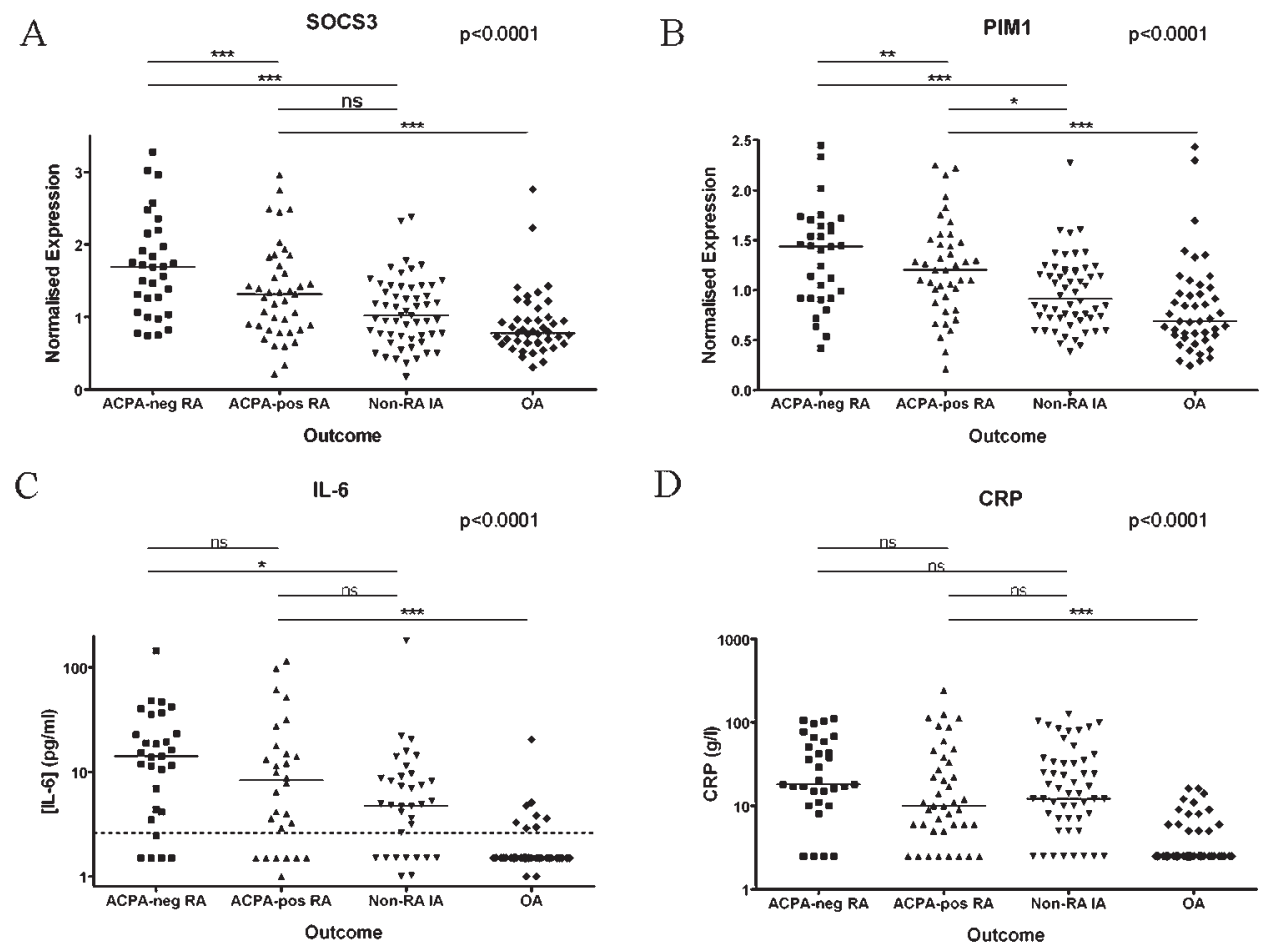

$\mathrm{D}$

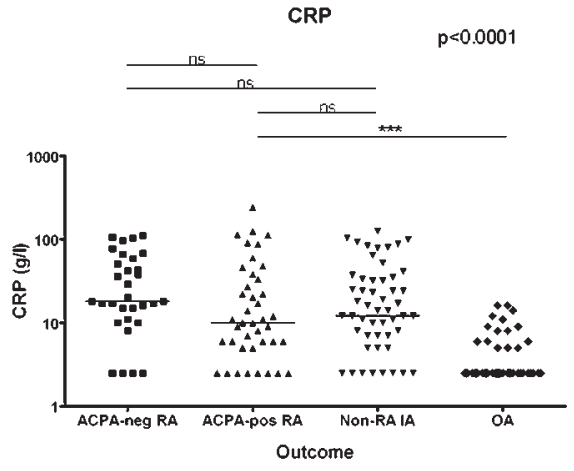

Figure 3 (A-B) Baseline profiles of indicated STAT3-regulated genes across four outcome groups; see online supplementary figure S4 and table S2 for additional examples and patient characteristics, respectively. (C-D) Baseline serum interleukin- $6(C ; n=131)$ and $C$ reactive protein $(D ; n=173)$ measurements across outcome groups. P-values: non-parametric analyses of variance (Kruskall-Wallis); for post-hoc analyses, 1, 2 and 3 asterisks denote $p<0.05,0.01$ and 0.001 , respectively (Dunn's multiple comparison analysis). ACPA, anti-citrullinated peptide antibodies; IL-6, interleukin 6; NS, not significant; RA, rheumatoid arthritis; IA, inflammatory arthritis.

Our observation that increased serum IL-6 levels among EAC attendees may predict a diagnosis of RA versus alternative arthritides is consistent with findings of previous biomarker studies, ${ }^{38} 39$ but ours is the first demonstration of a particular association with ACPA-negative disease (figure 3C). Striking correlations were seen between PB CD4 T-cell expression of several STAT3-inducible genes and paired, contemporaneous serum IL- 6 concentrations, which were independent of alternative acute phase markers (figures $4 \mathrm{~A}-\mathrm{D}$; also online supplementary figures S5A-D and table S6). STAT3 phosphorylation and downstream transcription is initiated by ligation of the cellsurface gp130 co-receptor by a range of ligands, including IL- $6 .{ }^{40}$ We measured IL- 6 in particular because of its recognised role as a pro-inflammatory cytokine in RA, ${ }^{41}$ and we excluded similar relationships with sIL6R (a surrogate of IL-6R trans-signalling) and other relevant substrates of STAT3 signalling. Therefore, the STAT3-inducible gene expression signature that we have identified does appear to be downstream of IL- 6 signalling. The capacity of IL- 6 alone to induce the STAT-3-regulated elements of our early RA gene expression signature in primary CD4 T cells was confirmed in vitro (online supplementary figures S6 and S7).

In conclusion, our data provide strong evidence for the induction of an IL-6-mediated STAT3 transcription programme in $\mathrm{PB}$ CD4 T cells of early RA patients, which is most prominent in ACPA-negative individuals and which contributes to a gene expression 'signature' that may have diagnostic utility. Furthermore, our findings could pave the way for a novel treatment paradigm, whereby emerging drugs targeting the IL-6-gp130-STAT3 'axis' ${ }^{42} 43$ find a rational niche as first choice agents in the management of ACPA-negative RA. Studies, such as ours, should ultimately contribute to the realisation of true 'personalised medicine' in early inflammatory arthritis, in which complex heterogeneity is stratified into pathophysiologically and therapeutically relevant subsets, with clear benefits in terms of clinical outcome and cost.

Contributors AGP was involved in the study's conception and design, sample and data collection; he conducted the majority of the laboratory work, performed a substantial part of the analysis and drafted the manuscript. DCS helped design the study and made a major contribution to data analysis, also contributing intellectually to revising the manuscript. SR made substantial contributions to the laboratory work, data analysis and manuscript revision. GW was involved in the study design and made a major contribution to the recruitment of patients, sample handling and the recording of clinical data, also critically appraising the manuscript draft. $\mathrm{CH}$ helped conceive and design the study, also contributing to the laboratory work and manuscript drafting. DAY helped conceive and design the study and performed some of the laboratory work; he also made a substantial contribution to data analysis, and contributed to manuscript drafting. JDI conceived and designed the study and contributed to data analysis; he provided intellectual input and supervision throughout the study and made a substantial contribution to manuscript drafting.

Acknowledgements AGP's work was supported by a clinical research fellowship from the Arthritis Research Campaign, UK. This work was supported by the UK NIHR Biomedical Research Centre for Ageing and Age-Related Disease Award to the Newcastle upon Tyne Hospitals NHS Foundation Trust. Clinical and translational research in the Musculoskeletal Research Group is supported by the Northumberland Tyne and Wear Comprehensive Local Research Network. The authors would like to thank the clinical staff at The Freeman Hospital who co-operated with the recruitment phase of the study, and, of course, the many patient volunteers who contributed so willingly.

Funding This study was supported by Arthritis Research UK (grant number 17983).

Competing interests None.

Ethics approval Ethics approval was provided by the Newcastle and North Tyneside Local Research Ethics Committee.

Provenance and peer review Not commissioned; externally peer reviewed. 

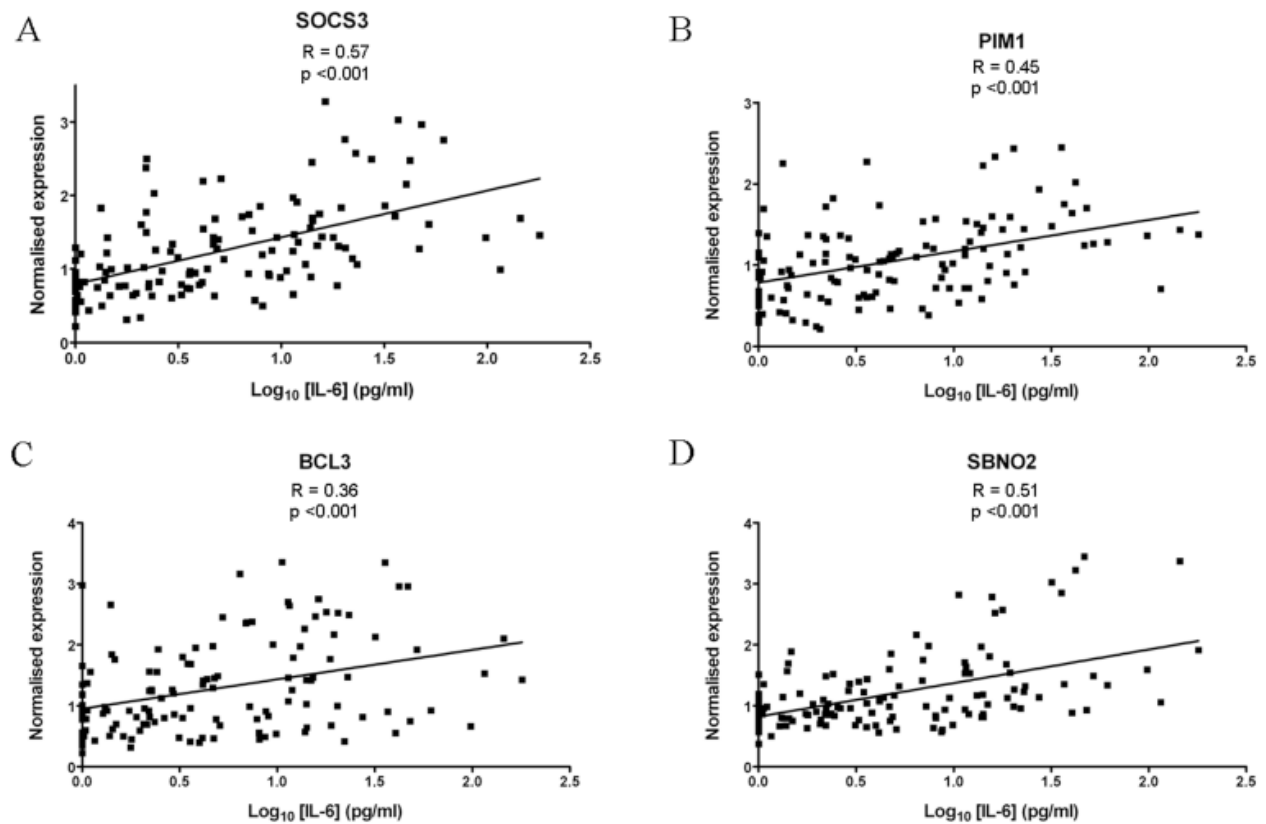

Figure 4 (A-D) Serum interleukin 6 (IL-6) concentrations correlate with STAT3-inducible gene expression in peripheral blood CD4 T cells (see online supplementary figure S5, for additional examples). Data are shown for 131 individuals in whom paired, contemporaneous samples were available at baseline; Pearson's R and associated $p$ values are shown.

Data sharing statement Raw and processed microarray data used in this study is available via Gene Expression Omnibus at: http://www.ncbi.nlm.nih.gov/geo/query/ acc.cgi?token $=$ bviftkociimgsnk\&acc $=$ GSE20 098 .

\section{REFERENCES}

1. Klareskog L, Catrina Al, Paget S. Rheumatoid arthritis. Lancet 2009;373:659-72.

2. Combe B, Landewe R, Lukas $C$, et al. EULAR recommendations for the management of early arthritis: report of a task force of the European Standing Committee for International Clinical Studies Including Therapeutics (ESCISIT). Ann Rheum Dis 2007;66:34-45.

3. van Gaalen FA, Linn-Rasker SP, van Venrooij WJ, et al. Autoantibodies to cyclic citrullinated peptides predict progression to rheumatoid arthritis in patients with undifferentiated arthritis: a prospective cohort study. Arthritis Rheum 2004;50:709-15.

4. Aletaha D, Neogi T, Silman AJ, et al. 2010 Rheumatoid arthritis classification criteria: an American College of Rheumatology/European League Against Rheumatism collaborative initiative. Arthritis Rheum 2010;62:2569-81.

5. van der Helm-van Mil AH, Detert J, le Cessie S, et al. Validation of a prediction rule for disease outcome in patients with recent-onset undifferentiated arthritis: moving toward individualized treatment decision-making. Arthritis Rheum 2008;58:2241-7.

6. Nishimura K, Sugiyama D, Kogata Y, et al. Meta-analysis: diagnostic accuracy of anti-cyclic citrullinated peptide antibody and rheumatoid factor for rheumatoid arthritis. Ann Intern Med 2007; 146:797-808.

7. Pratt AG, Isaacs JD, Wilson G. The clinical utility of a rule for predicting rheumatoid arthritis in patients with early undifferentiated arthritis: comment on the article by van der Helm-van Mil et al. Arthritis Rheum 2009;60:905; author reply 906.

8. Pascual V, Chaussabel D, Banchereau J. A genomic approach to human autoimmune diseases. Annu Rev Immunol 2010;28:535-71.

9. McKinney EF, Lyons PA, Carr EJ, et al. A CD8 + T cell transcription signature predicts prognosis in autoimmune disease. Nat Med 2010;16:586-91, 1p following 591.

10. van Baarsen LG, Bos WH, Rustenburg F, et al. Gene expression profiling in autoantibody-positive patients with arthralgia predicts development of arthritis. Arthritis Rheum 2010;62:694-704.

11. Batliwalla FM, Baechler EC, Xiao X, et al. Peripheral blood gene expression profiling in rheumatoid arthritis. Genes Immun 2005;6:388-97.

12. Lyons PA, Koukoulaki M, Hatton A, et al. Microarray analysis of human leucocyte subsets: the advantages of positive selection and rapid purification. BMC Genomics 2007;8:64.

13. McInnes IB, O'Dell JR. State-of-the-art: rheumatoid arthritis. Ann Rheum Dis 2010;69:1898-906.

14. Arnett FC, Edworthy SM, Bloch DA, et al. The American Rheumatism Association 1987 revised criteria for the classification of rheumatoid arthritis. Arthritis Rheum 1988;31:315-24.

15. Schroeder A, Mueller 0 , Stocker $S$, et al. The RIN: an RNA integrity number for assigning integrity values to RNA measurements. BMC Mol Biol 2006;7:3.
16. Johnson WE, Li C, Rabinovic A. Adjusting batch effects in microarray expression data using empirical Bayes methods. Biostatistics 2007;8:118-27.

17. Benjamini Y, Hochberg Y. Controlling the false discovery rate: a practical and powerful approach to multiple testing. J R Stat Soc Series B Stat Methodol 1995;57:289-300.

18. Cortes C. Vapnik V. Support-vector networks. Machine Learning 1995;20:273-97.

19. Livak KJ, Schmittgen TD. Analysis of relative gene expression data using real-time quantitative PCR and the 2(-Delta Delta C(T)) Method. Methods 2001;25:402-8.

20. Owaki T, Asakawa M, Morishima N, et al. STAT3 is indispensable to IL-27-mediated cell proliferation but not to IL-27-induced Th1 differentiation and suppression of proinflammatory cytokine production. J Immunol 2008;180:2903-11.

21. Starr $\mathbf{R}$, Willson TA, Viney EM, et al. A family of cytokine-inducible inhibitors of signalling. Nature 1997;387:917-21.

22. El Kasmi KC, Smith AM, Williams L, et al. Cutting edge: A transcriptional repressor and corepressor induced by the STAT3-regulated anti-inflammatory signaling pathway. (erratum appears in J Immunol 2008;180:3612 Note: Panopolous, Athanasia (corrected to Panopoulos, Athanasia D)). J Immunol 2007;179:7215-9.

23. Brocke-Heidrich $\mathbf{K}, \mathrm{Ge}$ B, Cvijic $\mathrm{H}$, et al. BCL3 is induced by IL-6 via Stat3 binding to intronic enhancer HS4 and represses its own transcription. Oncogene 2006;25:7297-304.

24. Richard M, Louahed J, Demoulin JB, et al. Interleukin-9 regulates NF-kappaB activity through BCL3 gene induction. Blood 1999;93:4318-27.

25. Gao J, McConnell MJ, Yu B, et al. MUC1 is a downstream target of STAT3 and regulates lung cancer cell survival and invasion. Int $\mathrm{J}$ Oncol 2009;35:337-45.

26. Akaishi H, Takeda K, Kaisho T, et al. Defective IL-2-mediated IL-2 receptor alpha chain expression in Stat3-deficient T lymphocytes. Int Immunol 1998;10:1747-51.

27. Matikainen S, Sareneva T, Ronni T, et al. Interferon-alpha activates multiple STAT proteins and upregulates proliferation-associated IL-2Ralpha, c-myc, and pim-1 genes in human T cells. Blood 1999;93:1980-91.

28. Nichane M, Ren X, Bellefroid EJ. Self-regulation of Stat3 activity coordinates cellcycle progression and neural crest specification. EMBO J 2010;29:55-67.

29. Hirano T, Ishihara K, Hibi M. Roles of STAT3 in mediating the cell growth, differentiation and survival signals relayed through the IL-6 family of cytokine receptors. Oncogene 2000;19:2548-56.

30. Nowell MA, Williams AS, Carty SA, et al. Therapeutic targeting of IL-6 trans signaling counteracts STAT3 control of experimental inflammatory arthritis. J Immunol 2009;182:613-22

31. Rose-John S, Scheller J, Elson G, et al. Interleukin-6 biology is coordinated by membrane-bound and soluble receptors: role in inflammation and cancer. J Leukoc Biol 2006;80:227-36.

32. Eyles JL, Hickey MJ, Norman MU, et al. A key role for G-CSF-induced neutrophil production and trafficking during inflammatory arthritis. Blood 2008;112:5193-201. 
33. Rho YH, Solus J, Sokka T, et al. Adipocytokines are associated with radiographic joint damage in rheumatoid arthritis. Arthritis Rheum 2009;60:1906-14.

34. El Kasmi KC, Smith AM, Williams L et al. Cutting edge: A transcriptional repressor and corepressor induced by the STAT3-regulated anti-inflammatory signaling pathway. (Erratum appears in J Immunol 2008;180:3612 Note: Panopolous, Athanasia (corrected to Panopoulos, Athanasia D)). J Immunol 2007;179:7215-9.

35. Altman DG, Bland JM. Diagnostic tests 2: Predictive values. BMJ 1994;309:102.

36. Ponchel F, Morgan AW, Bingham SJ, et al. Dysregulated lymphocyte proliferation and differentiation in patients with rheumatoid arthritis. Blood 2002;100:4550-6.

37. Goronzy JJ, Weyand CM. Rheumatoid arthritis. Immunol Rev 2005;204:55-73.

38. Kokkonen H, Söderström I, Rocklöv J, et al. Up-regulation of cytokines and chemokines predates the onset of rheumatoid arthritis. Arthritis Rheum 2010;62:383-91.
39. Karlson EW, Chibnik LB, Tworoger SS, et al. Biomarkers of inflammation and development of rheumatoid arthritis in women from two prospective cohort studies. Arthritis Rheum 2009;60:641-52.

40. Schindler CW. Series introduction. JAK-STAT signaling in human disease. $J$ Clin Invest 2002:109:1133-7.

41. Fonseca JE, Santos MJ, Canhão H, et al. Interleukin-6 as a key player in systemic inflammation and joint destruction. Autoimmun Rev 2009;8:538-42.

42. Nishimoto N, Miyasaka N, Yamamoto K, et al. Long-term safety and efficacy of tocilizumab, an anti-LL-6 receptor monoclonal antibody, in monotherapy, in patients with rheumatoid arthritis (the STREAM study): evidence of safety and efficacy in a 5-year extension study. Ann Rheum Dis 2009;68:1580-4.

43. Cohen S, Fleischmann R. Kinase inhibitors: a new approach to rheumatoid arthritis treatment. Curr Opin Rheumatol 2010;22:330-5. 